\title{
Rho signaling pathway enhances proliferation of PASMCs by suppressing nuclear translocation of Smad1 in PAH
}

\author{
HONGWEI WEI ${ }^{*}$, DONGQING ZHANG ${ }^{2 *}$, LILI LIU $^{2}$, WEI XIA ${ }^{2}$ and FUHAI LI ${ }^{2}$ \\ ${ }^{1}$ Department of Pediatrics, The Third Hospital of Jinan, Jinan, Shandong 250132; \\ ${ }^{2}$ Department of Pediatrics, Qilu Hospital of Shandong University, Jinan, Shandong 250012, P.R. China
}

Received December 28, 2017; Accepted July 26, 2018

DOI: $10.3892 /$ etm.2018.6942

\begin{abstract}
Bone morphogenetic protein (BMP) and Rho kinase signaling pathways exert counter regulatory effects on pulmonary artery smooth muscle cell (PASMC) proliferation in pulmonary artery hypertension (PAH). To elucidate the mechanism of this interaction, the present study tested whether Rho kinase activated by platelet derived growth factor-BB (PDGF-BB) enhances PASMC proliferation by suppressing the nuclear translocation of Smad1 induced by BMP-2. BMP-2 was used to activate the Smad1 signaling pathway and PDGF-BB was used to activate the Rho kinase signaling pathway when cells were pretreated with or without Rho-associated protein kinase (ROCK) inhibitor Y-27632 or dual specificity mitogen-activated protein kinase kinase (MEK) 1 and 2 inhibitor U0126. Western blotting was used to determine the expression of the components of the Rho signaling pathway, and the expression of various variants of phosphorylated mothers against decapentaplegic homolog ( $\mathrm{p}-\mathrm{Smad}) 1$ in the cytoplasm and nucleus. Immunofluorescent staining was used to observe subcellular distribution of p-Smad1. A cell counting kit was used to analyze cell proliferation. Active RhoA/Rho kinase signaling and decreased nuclear translocation of Smad1 were found in primary cultured PASMCs from the rat model of PAH compared with the control PASMCs. Treatment with BMP-2 significantly increased nuclear accumulation of Smad1 and inhibited the proliferation of PASMCs. However, pretreatment with PDGF-BB significantly decreased the nuclear accumulation of Smad1 induced by BMP-2 and enhanced the proliferation of PASMCs. Furthermore, pretreatment with Y-27632 or U0126 was found to restore the nuclear translocation
\end{abstract}

Correspondence to: Professor Fuhai Li, Department of Pediatrics, Qilu Hospital of Shandong University, 107 Wenhua Xi Road, Jinan, Shandong 250012, P.R. China

E-mail: fuhai_li@163.com

*Contributed equally

Key words: pulmonary artery smooth muscle cell, pulmonary artery hypertension, Rho signaling, bone morphogenetic protein 2 , mothers against decapentaplegic homolog 1 of Smad1 suppressed by PDGF-BB and decrease the proliferation of PASMCs. In conclusion, the present study suggested that Rho kinase activated by PDGF-BB suppressed BMP-2-induced nuclear translocation of Smad1 via the MEK/mitogen-activated protein kinase and enhanced BMP-2-inhibited proliferation of PASMCs.

\section{Introduction}

BMPs are a group of multi-functional growth factors of the transforming growth factor $\beta$ (TGF- $\beta$ ) superfamily. Binding of BMP to BMP receptor type-2 (BMPR2) leads to phosphorylation of BMPR1A, which activates its kinase activity and phosphorylates regulatory Smads (R-Smads), including Smad1 at Ser463 and Ser465 (1). Phosphorylated R-Smads (p-Smads) form complexes with common-partner Smads and translocate into the nucleus to regulate transcription of target genes (2).

Loss of function and mutations in the BMPR2 gene have been identified in $\sim 80 \%$ of patients with familial pulmonary artery hypertension $(\mathrm{PAH})$ and in $11-40 \%$ of patients with sporadic PAH (3-5). Reduced expression levels of BMPR1A and BMPR2 were also observed in the lungs of patients with idiopathic and secondary PAH without detectable BMPR2 mutations $(6,7)$ and in experimental animal models of PAH $(8,9)$. Expression and phosphorylation levels of Smad1 significantly decreased in lungs of rats with monocrotaline (MTC)-induced PAH $(10,11)$. The above evidence suggests that the BMP receptor signaling is associated with the pathogenesis of PAH. However, it remains to be elucidated how the BMP receptor signaling causes PAH.

Smads have highly conserved $\mathrm{N}$ - and C-terminal regions, termed mad homology ( $\mathrm{MH}) 1$ and $\mathrm{MH} 2$ domains, respectively (12). The MH1 and MH2 domains are bridged by a linker region with a variable length and consensus amino acid sequences, which may be phosphorylated at Ser206 by mitogen-activated protein kinase (MAPK) (13). The phosphorylation of Ser206 recruits E3 ubiquitin-protein ligase SMURF1 to the linker region and leads to degradation of Smad1 and interruption of nuclear translocation $(12,14)$. Activated MAPK signaling has been observed to be positively associated with the occurrence and development of PAH. However, it remains to be elucidated whether MAPK contributes to PAH by promoting the degradation of Smad1 $(15,16)$. Previous studies suggested the involvement of the RhoA/Rho kinase pathway in the 
pathogenesis of PAH induced by hypoxia, monocrotaline or high blood flow (17-19). MAPK is one of the downstream signaling molecules of the RhoA/Rho kinase pathway $(20,21)$. The authors of the present study hypothesized that the RhoA/Rho kinase signaling pathway may promote the process of PAH by attenuating the BMP signaling pathway.

In the present study, using an experimental rat model of PAH, a primary culture of PASMCs was established and used to analyze cell proliferation under differential drug treatment. The present study aimed to establish whether the RhoA/Rho kinase signaling pathway activated by PDGF-BB enhances the proliferation of PASMCs by suppressing the nuclear translocation of Smad1 induced by BMP-2.

\section{Materials and methods}

Primary PASMC isolation and culture. A total of 30 Male Sprague-Dawley (SD) rats (age, 4 weeks; weight 150-200 g) were purchased from Shanghai SLAC Laboratory Animal Co., Ltd., (Shanghai, China). Animals were housed under standard environmental conditions with a regulated temperature $\left(20-24^{\circ} \mathrm{C}\right)$ and humidity (45-50\%), with a $12 \mathrm{~h}$ light/dark cycle and free access to food and water. Animals were then divided into two groups $(n=15)$. One group were subcutaneously injected with a single dose of MCT (40 mg/kg body weight; Sigma-Aldrich; Merck KGaA, Darmstadt, Germany) to induce PAH as previously reported (22). The second group were subcutaneously injected with an equal volume of normal saline as a control and then fed normally for two weeks. The control SD rats and PAH rats were subsequently anesthetized and pulmonary artery systolic pressure was measured, as previously described, to determine the establishment of the PAH model (23). All rats were sacrificed and the lungs were harvested. Animal welfare and experimental procedures were carried out in accordance with the Guide for the Care and Use of Laboratory Animals, published by the Ministry of Science and Technology of China (24), and were approved by the Animal Ethics Committee of Shandong University.

Intralobar pulmonary arteries (2nd branches; 100-400 $\mu \mathrm{m}$ diameter) were dissected, rinsed in cold PBS two times, and the adventitia and endothelium were removed using scissors and a cotton swab in PBS. Arteries were cut into small pieces and dissociated in $0.25 \%$ pancreatin solution (Gibco; Thermo Fisher Scientific, Inc., Waltham, MA, USA) at $37^{\circ} \mathrm{C}$ for $30 \mathrm{~min}$. The dissociation solution was removed following centrifugation at a speed of $200 \mathrm{x} \mathrm{g}$ for $5 \mathrm{~min}$ at $4^{\circ} \mathrm{C}$, and $5 \mathrm{ml}$ Dulbecco's Modified Eagle's medium (DMEM; Gibco; Thermo Fisher Scientific, Inc.) was added to re-suspend the pellets.

Collected cells were cultured in $25 \mathrm{ml}$ culture flasks for 3-5 days in 10\% fetal bovine serum (FBS)-DMEM in a humidified atmosphere of $5 \% \mathrm{CO}_{2}$ at $37^{\circ} \mathrm{C}$. Purity of the cultures was assessed by morphological appearance using phase-contrast microscopy (magnification, x200) and immunofluorescent staining using selective anti- $\alpha$-smooth muscle actin (Santa Cruz Biotechnology, Inc., Dallas, TX, USA) antibody observed under a fluorescence microscope at a magnification of x200. Cells were used for experiments at passage 3-5.

PASMC treatment. PASMCs were cultured in $10 \% \mathrm{FBS}$ $\mathrm{DMEM}$ at $37^{\circ} \mathrm{C}$ in a humidified atmosphere of $5 \% \mathrm{CO}_{2}$ to $95 \%$ confluence. The medium was changed to $0.1 \%$ FBS-DMEM for $24 \mathrm{~h}$ and, subsequently, fresh 10\% FBS-DMEM was added to synchronize the cell cycle to the $\mathrm{G}_{0}$ phase. Rho, in combination with GTP, activates downstream Rho-associated protein kinase (ROCK), phosphorylating its downstream substrates (25). PASMCs from the control rats were pretreated with the specific drug Y-27632 (10 $\mu \mathrm{M}$; Sigma-Aldrich; Merck KGaA) of ROCK inhibitor, or U0126 (20 $\mu \mathrm{M}$; Sigma-Aldrich; Merck KGaA) of MEK1/2 inhibitor, were added $12 \mathrm{~h}$ prior to PDGF-BB $(10 \mathrm{ng} / \mathrm{ml}$; PeproTech, Inc., Rocky Hill, NJ, USA) treatment to activate the Rho kinase. PASMCs from PAH rats were pretreated $12 \mathrm{~h}$ prior to PDGF-BB or BMP-2 (2 ng/ml; PeproTech, Inc., Rocky Hill, NJ, USA) treatment with the specific drugs Y-27632 or U0126 to activate Smad1.

Immunofluorescence staining. PASMCs from PAH rats at different time-points following treatment (0-210 min) were fixed in 4\% paraformaldehyde at room temperature for $20 \mathrm{~min}$ and permeabilized in $0.1 \%$ Triton $\mathrm{X}-100$ in PBS at room temperature for $20 \mathrm{~min}$. Samples were then blocked with $5 \%$ BSA at room temperature for $30 \mathrm{~min}$. The fixed cells were incubated with rabbit-phospho-Smad1 (phosphorylation site, Ser463/465; 1:1,000; cat. no. 13820; Cell Signaling Technology, Inc., Danvers, MA, USA) primary antibodies at $4^{\circ} \mathrm{C}$ overnight and a Rhodamine Red-labeled goat anti-rabbit immunoglobulin G (1:2,000; cat. no. 305-295-003; Jackson ImmunoResearch Laboratories, Inc., West Grove, PA, USA) secondary antibody at room temperature for $60 \mathrm{~min}$ to assess nuclear translocation of p-Smad1 using confocal fluorescence microscopy (magnification, $\mathrm{x} 200$ ). The nuclei were counterstained with DAPI (Roche Diagnostics, Basel, Switzerland) at room temperature for $3 \mathrm{~min}$. A total of 10 images per group at each time point were randomly selected to observe nuclear translocation and calculated the percentage of cells with p-Smad1 (Ser463/465) nuclear staining using Image J software (National Institute of Health, Bethesda, MD, USA).

Western blotting. Nuclear proteins were isolated using nuclear protein extraction kit (Bestbio Corporation, Nanjing, China) and the total cellular proteins were extracted using total protein extraction kit (Bestbio Corporation, Nanjing, JS, CN). Protein concentration was detected using a BCA protein assay kit (Thermo Fisher Scientific, Inc.). A total of $30 \mu \mathrm{g}$ protein was loaded per lane and subjected to $12 \%$ SDS-PAGE. Samples were then electrophoretically transferred to nitrocellulose membranes. Following blocking with $5 \% \mathrm{BSA}$ at room temperature for $30 \mathrm{~min}$, the membranes were incubated with the following primary antibodies at $4{ }^{\circ} \mathrm{C}$ overnight: Rabbit-ROCK1 (cat. no. 4035), mouse-MEK1 (cat. no. 2352), rabbit-phospho-MEK1/2 (Ser217/221; cat. no. 9154), mouse-ERK1/2 (cat. no. 4696), rabbit-phospho-ERK1/2 (Thr202/Tyr204; cat. no. 4376), rabbit-phospho-Smad1 (Ser206; cat. no. 9553), rabbit-phospho-Smad1 (Ser463/465; cat. no. 13820), rabbit-Smad1 (cat. no. 9743; 1:1,000; all Cell Signaling Technology, Inc.), mouse- $\beta$-actin (1:1,000 dilution; cat. no. 21800; Signalway Antibody LLC, College Park, MD, USA) or rabbit-histone H3.1 (1:500; cat. no. 32667; Signalway Antibody LLC). Subsequently, the membranes were incubated with horseradish peroxidase-conjugated goat anti-mouse immunoglobulin G (cat. no. 115-005-166) or goat anti-rabbit 

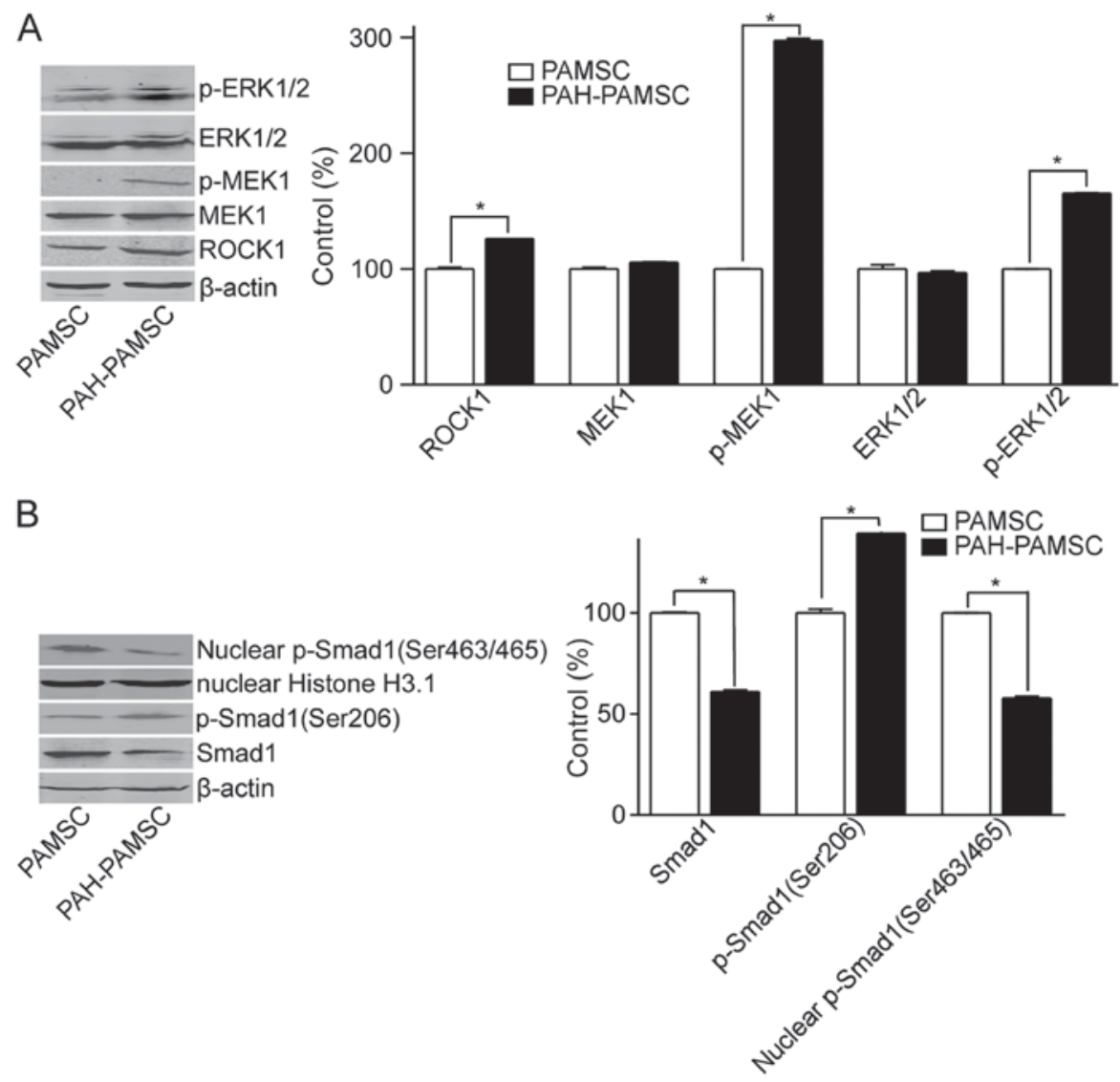

Figure 1. Expression levels of Smad1 and proteins associated with the Rho signaling pathway. PASMCs were isolated from pulmonary arteries of control rats or rat models of PAH and cultured. Total and nuclear proteins were extracted. (A) Protein levels of p-ERK1/2, ERK1/2, p-MEK1, MEK1 and ROCK1 in PASMCs and PAH-PASMCs were measured by western blotting. (B) Total Smad1, p-Smad1 (Ser206) and nuclear Smad1 (Ser463/465) protein expression levels were measured by western blotting in PASMCs and PAH- PASMCs. " $\mathrm{P}<0.05$. Data are presented as the mean \pm standard deviation, $\mathrm{n}=3$. PAH, pulmonary artery hypertension; ROCK1, Rho-associated protein kinase 1; p, phosphorylated; PASMC, pulmonary artery smooth muscle cell; ERK1/2, mouse-mitogen-activated protein kinase; MEK1, dual specificity mitogen-activated protein kinase kinase; Smad1, mothers against decapentaplegic homolog 1.

immunoglobulin G (cat. no. 111-005-144; both 1:2,000; Jackson ImmunoResearch Laboratories, Inc.) at room temperature for $60 \mathrm{~min}$. Bands were identified through enhanced chemiluminescence (ECL) using the Immobilon ${ }^{\mathrm{TM}}$ Western Chemiluminescent HRP Substrate (EMD Millipore, Billerica, MA, USA). Scanned images were inverted and quantified by densitometric analysis with Quantity One 1-D software (version 4.6.9; Bio-Rad Laboratories, Inc., Hercules, CA, USA). Histone $\mathrm{H} 3.1$ and $\beta$-actin were used as loading controls for nuclear and total cellular proteins, respectively.

Cell proliferation analyses. Cell proliferation was analyzed using Cell Counting kit (Dojindo Molecular Technologies, Inc., Kumamuto, Japan) according to the manufacturer's protocol. Briefly, PAH-PASMCs were cultured in a 96-well plate overnight and were pre-treated with the specific drug (Y-27632 or U0126) $12 \mathrm{~h}$ prior to the PDGF-BB or BMP-2 treatment for $24 \mathrm{~h}$. A total of $10 \mu \mathrm{l}$ of the WST-8 solution from the cell counting kit was added to each well and incubated for another $4 \mathrm{~h}$. Cell viability was analyzed by measuring the absorbance at a wavelength of $570 \mathrm{~nm}$ using a microplate reader. The optical density value was considered proportional to the number of living cells.

Statistical analysis. Data are presented as the mean \pm standard deviation, one-way analysis of variance followed by a post-hoc
Bonferroni's test was performed using SPSS software (version 16.0; SPSS, Inc., Chicago, IL, USA) to compare the differences between groups. $\mathrm{P}<0.05$ was considered to indicate a statistically significant difference.

\section{Results}

RhoA/Rho signaling pathway is involved in PAH through regulating the phosphorylation and degradation of Smadl. To study the alterations in the RhoA/Rho and BMP signaling in PAH, the protein expression levels of ROCK1, MEK1 and ERK, as well as total Smad1 and p-Smad1 in PASMCs from the control and PAH rats were determined. The expression levels of ROCK1 increased in PASMCs from the PAH group compared with the control rats (Fig. 1A). The same effect was observed for the phosphorylated forms of MEK1, ERK and Smad1 (Ser206), but not the total proteins (Fig. 1A). The increased phosphorylation of Smad1 at Ser206 and decreased expression levels of Smad1 were observed in PAH-PASMC compared with the control PASMCs (Fig. 1B). By contrast, the nuclear expression levels of p-Smad1 (Ser463/465), which stimulates transcription of target genes, decreased in PASMCs from the rat models of PAH compared with the control rats (Fig. 1B). These results indicate that the Rho signaling pathway was activated in PAH compared with the control group, leading 


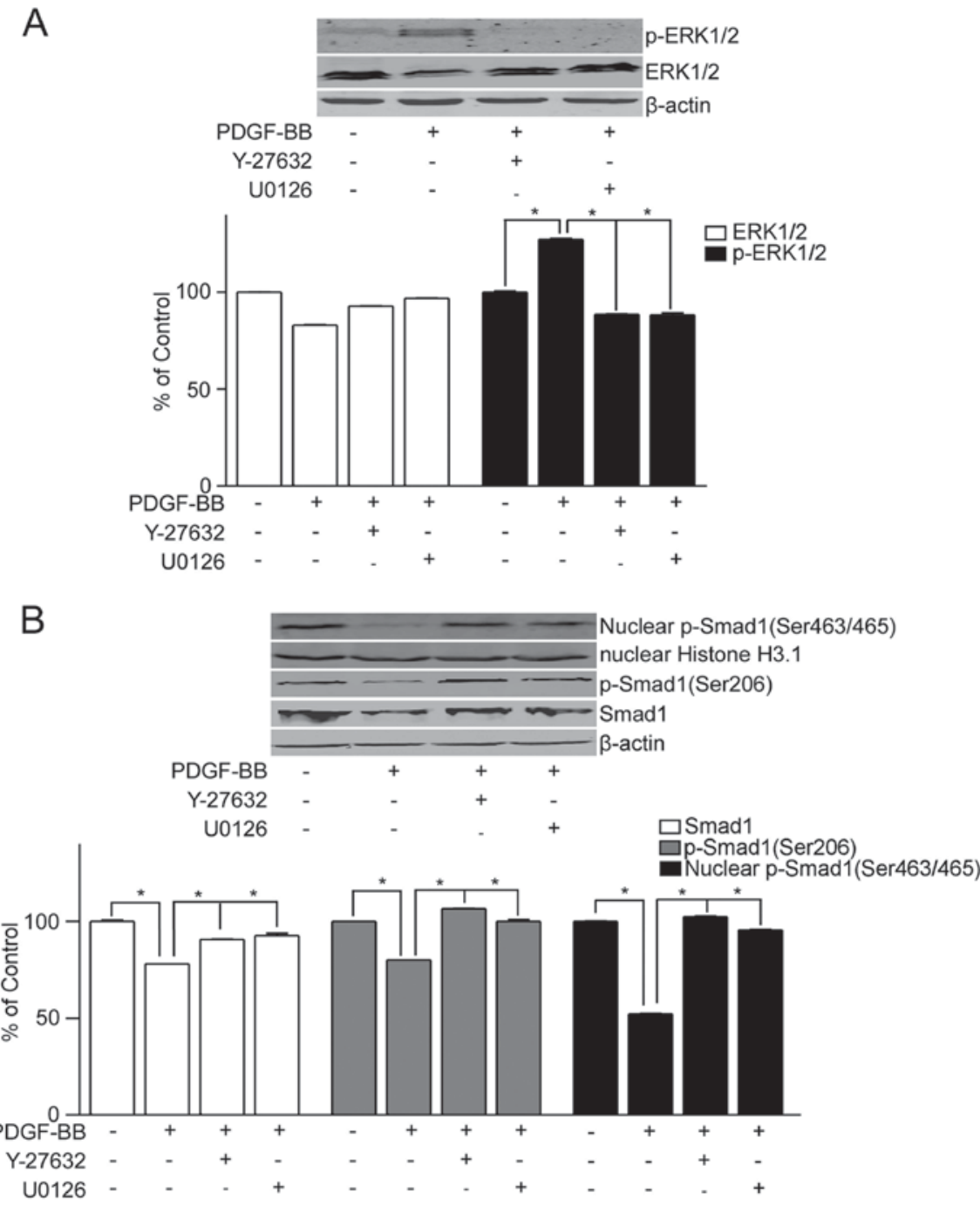

Figure 2. Rho signaling pathway affects Smad1 in an in vitro model of pulmonary artery hypertension. PASMCs were treated with $10 \mathrm{ng} / \mathrm{ml} \mathrm{Rho}$ activator PDGF-BB, $10 \mu \mathrm{M}$ ROCK1 inhibitor Y-27632 and $20 \mu \mathrm{M}$ MEK1 inhibitor U0126. After $12 \mathrm{~h}$, total and nuclear proteins were extracted. (A) ERK1/2 and p-ERK1/2 protein expression levels affected by the activation and inhibition of the Rho signaling pathway in PASMCs were measured by western blotting. (B) Total Smad1, p-Smad1 (Ser206) and nuclear Smad1 (Ser463/465) protein levels affected by the activation and inhibition of the Rho signaling pathway in PASMCs were measured by western blotting. Data are presented as the mean \pm standard deviation, $n=3$. ${ }^{*} \mathrm{P}<0.05$. ERK $1 / 2$, Smad1, mouse-mitogen-activated protein kinase; p, phosphorylated; mothers against decapentaplegic homolog 1; PDGF-BB, platelet derived growth factor-BB; PASMC, pulmonary artery smooth muscle cell.

to the degradation and decreased translocation of Smad1 into the nucleus.

To further confirm the role of the RhoA/Rho signaling in the regulation of Smad1, the PASMCs from control rats were treated with PDGF-BB, with or without Y-27632 or U0126 (the inhibitors of ROCK or MEK1/2, respectively) to activate or inhibit the RhoA/Rho signaling, respectively. Phosphorylation of ERK increased following treatment with PDGF-BB compared with the untreated control, but not following co-treatment with Y-27632 or U0126 (Fig. 2A). Furthermore, decreased expression levels of Smad1 and p-Smad1 (Ser206) were observed in PASMCs treated with PDGF-BB compared with the untreated control, but not in cells treated with PDGF-BB + Y-27632 or U0126 (Fig. 2B). Decreased expression levels of nuclear p-Smad1 (Ser463/465) were also observed in PASMCs treated with PDGF-BB compared with the untreated control cells, but not in PASMCs treated with PDGF-BB + Y-27632 or U0126 (Fig. 2B).
Taken together, the above data indicated that activation of the RhoA/Rho signaling may lead to the degradation of Smad1 and the decrease in the nuclear translocation of p-Smad1, suggesting that the RhoA/Rho signaling may be involved in PAH through regulating Smad1.

RhoA/Rho activation inhibits BMP-2-induced nuclear translocation of Smad1. To determine the dynamic interactions between the RhoA/Rho and BMP-2 signaling, immunofluorescent staining of $\mathrm{p}$-Smad1 was performed in PAH-PASMCs under various drug treatments. Treatment with BMP-2 significantly increased the levels of nuclear p-Smad1 (Ser463/465), which was observed from 30 to 120 min after treatment with BMP-2 compared with the untreated PAH-PASMCs, the maximum increase was at $60 \mathrm{~min}$ and markedly decreased at $150 \mathrm{~min}$ following treatment with BMP-2 for $210 \mathrm{~min}$ (Fig. 3A and B). Furthermore, an increase in the expression of p-Smad1 (Ser463/465) and p-Smad1 (Ser206), but not the total 

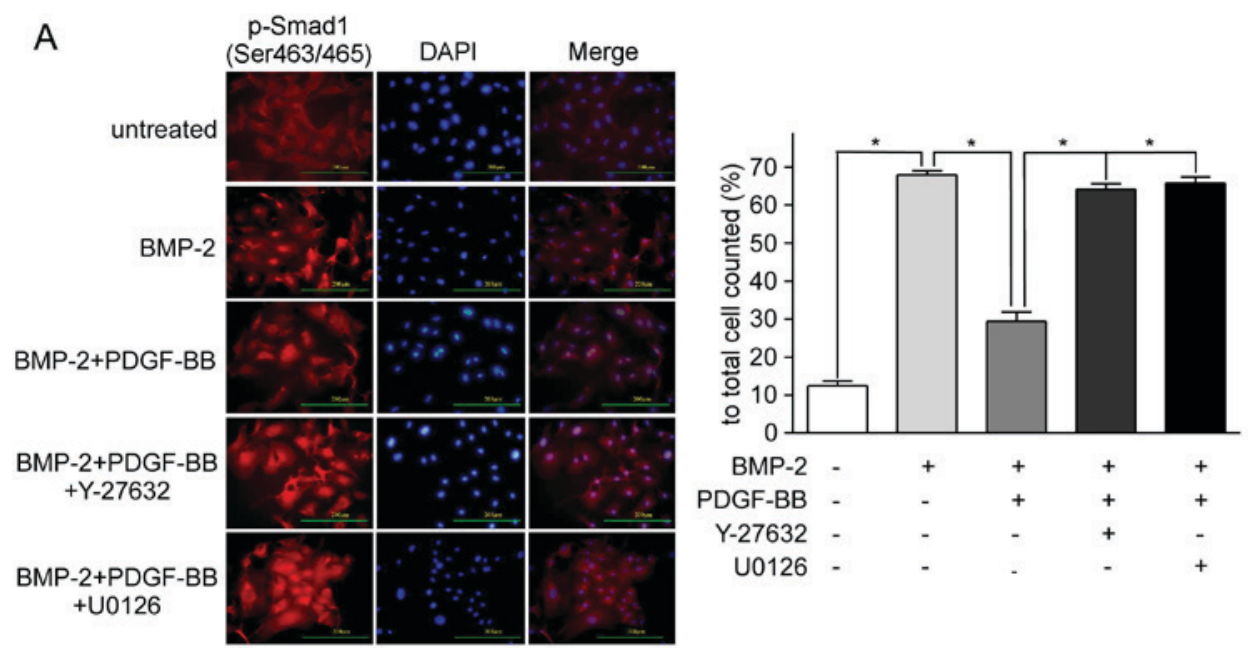

B

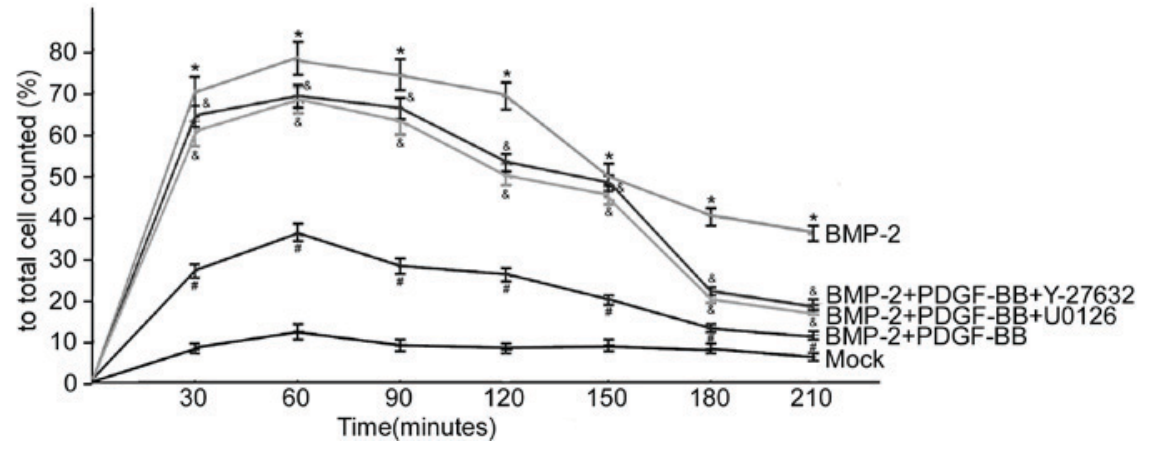

C
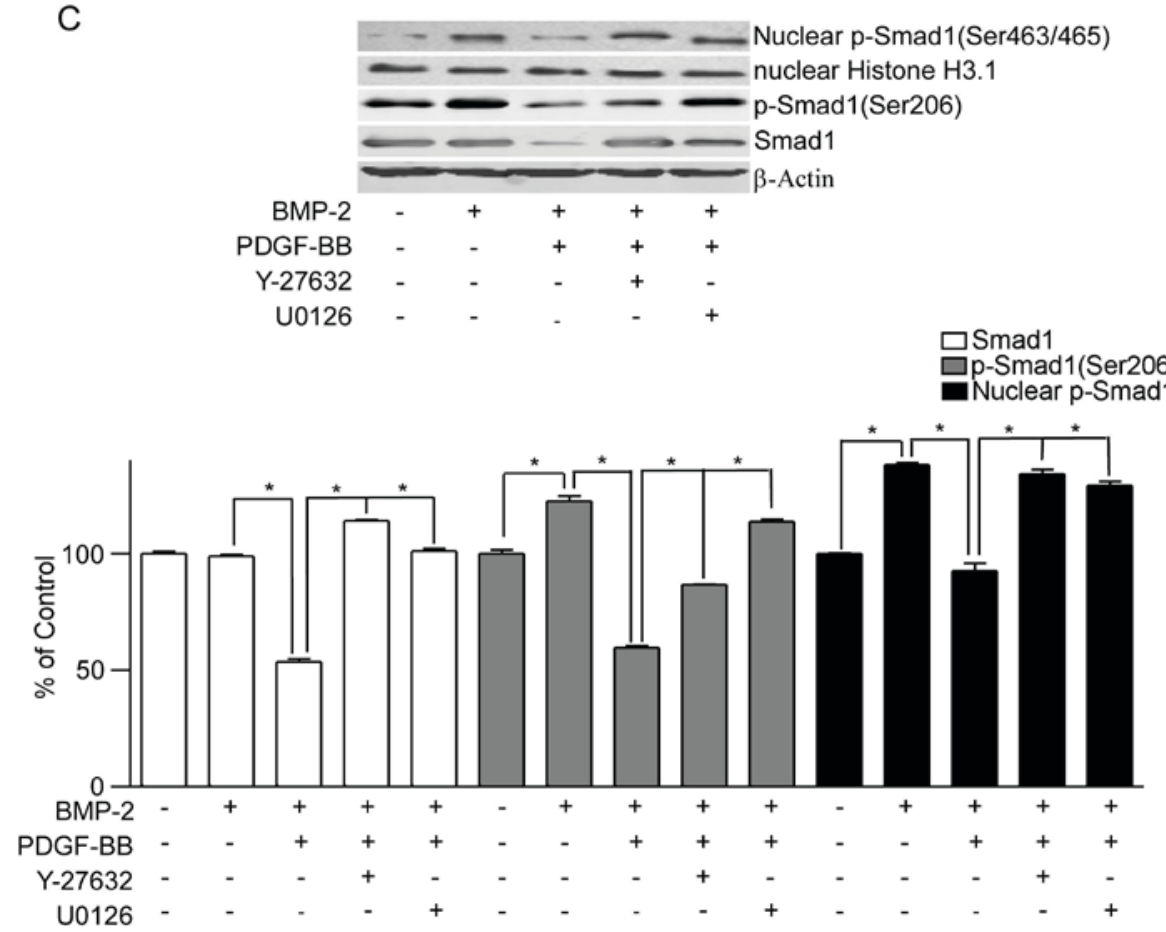

Figure 3. Activation of the Rho signaling pathway inhibits BMP-2-induced Smad1 expression in PAH. PAH-PASMCs were cultured and treated with $10 \mathrm{ng} / \mathrm{ml}$ Rho activator PDGF-BB, $2 \mathrm{ng} / \mathrm{ml}$ Smad1 activitor BMP-2, $10 \mu \mathrm{M}$ ROCK inhibitor Y-27632 and $20 \mu \mathrm{M}$ MEK1 inhibitor U0126. Immunofluorescence staining results were measured at 0-210 min of the experiment. Or $12 \mathrm{~h}$ following treatment with final BMP-2, total and nuclear proteins were extracted, respectively. (A) Representative confocal immunofluorescent staining micrographs of distribution of p-Smad1 (Ser463/465) 60 min after exposure to activator and inhibitor in PASMCs. The results were analyzed quantitatively. "P<0.05; Scale bar, $200 \mu \mathrm{m}$. (B) Changes of the percentage of cells positive for p-Smad (Ser463/465) nuclear accumulation after activator and inhibitor exposure in PAH-PASMCs. ${ }^{*} \mathrm{P}<0.05$. the BMP-2 group vs. the untreated group; ${ }^{~} \mathrm{P}<0.05$ the BMP-2+PDGF-BB group vs. the BMP-2 group; ${ }^{\text {P }}<0.05$ the BMP-2+PDGF-BB+Y27632 or the BMP-2+PDGF-BB+U0126 group vs. the BMP-2+PDGF-BB group. (C) Total Smad1, total p-Smad1 (Ser206) and nuclear p-Smad1 (Ser463/465) protein levels following activation of the Rho and BMP-2 signaling pathways in PASMCs from rat models of PAH were measured by western blotting. ${ }^{*} \mathrm{P}<0.05$. Data are presented as the mean \pm standard deviation, $\mathrm{n}=3$. PAH, pulmonary artery hypertension; PASMC, pulmonary artery smooth muscle cell; BMP-2, bone morphogenetic protein 2; PDGF-BB, platelet derived growth factor-BB; Smad1, mothers against decapentaplegic homolog 1; p, phosphorylated. 
Smad1 protein was observed in the cytoplasm and nucleus of the PAH-PASMCs treated with BMP-2, compared with untreated PAH-PASMCs (Fig. 3C). Pretreatment with PDGF-BB (12 h before adding BMP-2) decreased the magnitude of nuclear expression of p-Smad1 (Ser463/465) induced by BMP-2 (Fig. 3A and B), as well as decreased the expression of p-Smad1 (Ser206) and total Smad1 protein, compared with PAH-PASMCs treated with BMP-2 (Fig. 3C). Furthermore, pretreatment with Y-27632 or U0126 (12 h before treatment with PDGF-BB) rescued the BMP-2 induced nuclear translocation of p-Smad1 (Ser463/465) that was inhibited by PDGF-BB (Fig. 3A-C). In addition, Y-27632 and U0126 rescued the PDGF-BB-decreased expression of p-Smad1 (Ser206) and total Smad1 (Fig. 3C).

RhoA/Rho activation rescues BMP-2-inhibited proliferation of PAH-PASMCs. Compared with the untreated PAH-PASMCs, treatment with BMP-2 significantly decreased the viability of PAH-PASMCs (Fig. 4). Pretreatment with PDGF-BB (12 h after adding BMP-2) for $12 \mathrm{~h}$ rescued the decreased viability of PAH-PASMCs (Fig. 4). Furthermore, pretreatment (12 h before adding PDGF-BB) with ROCK and ERK1/2 inhibitors, Y-27632 and U0126, counteracted the effect of PDGF-BB; the viability of PAH-PASMCs decreased to the comparable level to the group of cells treated with BMP-2 only (Fig. 4).

\section{Discussion}

The results of the present study indicated that activation of the Rho signaling pathway decreased Smad1 and nuclear translocation of p-Smad1 in PAH-PASMCs. PDGF-BB, an activator of the Rho signaling pathway, interfered with the

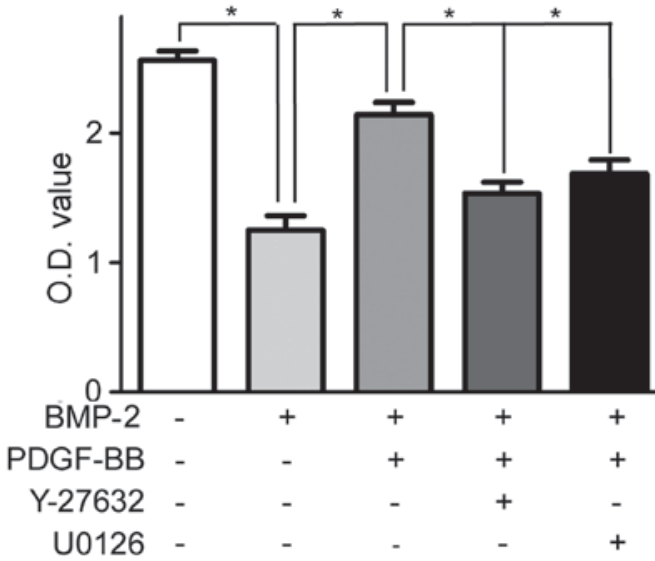

Figure 4. Activation of the Rho signaling pathway promotes proliferation of PAH-PASMCs inhibited by BMP-2. PAH-PASMCs were cultured and treated with $10 \mathrm{ng} / \mathrm{ml}$ Rho activator PDGF-BB, $2 \mathrm{ng} / \mathrm{ml}$ Smad1 activitor BMP-2, $10 \mu \mathrm{M}$ ROCK1 inhibitor Y-27632 and $20 \mu \mathrm{M}$ MEK1 inhibitor U0126. $24 \mathrm{~h}$ following treatment with final BMP-2, the proliferation of PAH-PASMCs affected by the activation of the Rho kinase and BMP-2 signaling pathways was measured by a WST- 8 assay. ${ }^{*} \mathrm{P}<0.05$. Data are presented as the mean \pm standard deviation, $\mathrm{n}=3$. PASMC, pulmonary artery smooth muscle cell; PAH, pulmonary artery hypertension; PAH-PASMCs, PASMCs from rat models of PAH; BMP-2, bone morphogenetic protein 2; PDGF-BB, platelet derived growth factor-BB.

BMP-2-stimulated nuclear translocation of $\mathrm{p}$-Smad1 via a Rho/ROCK/MEK/ERK-dependent mechanism (Fig. 5). PDGF-BB stimulated PASMC proliferation inhibited by BMP-2.

The present study demonstrated that PDGF-BB induced degradation of Smad1 through phosphorylation of Smad1 at Ser206, which reduced the nuclear translocation of this protein

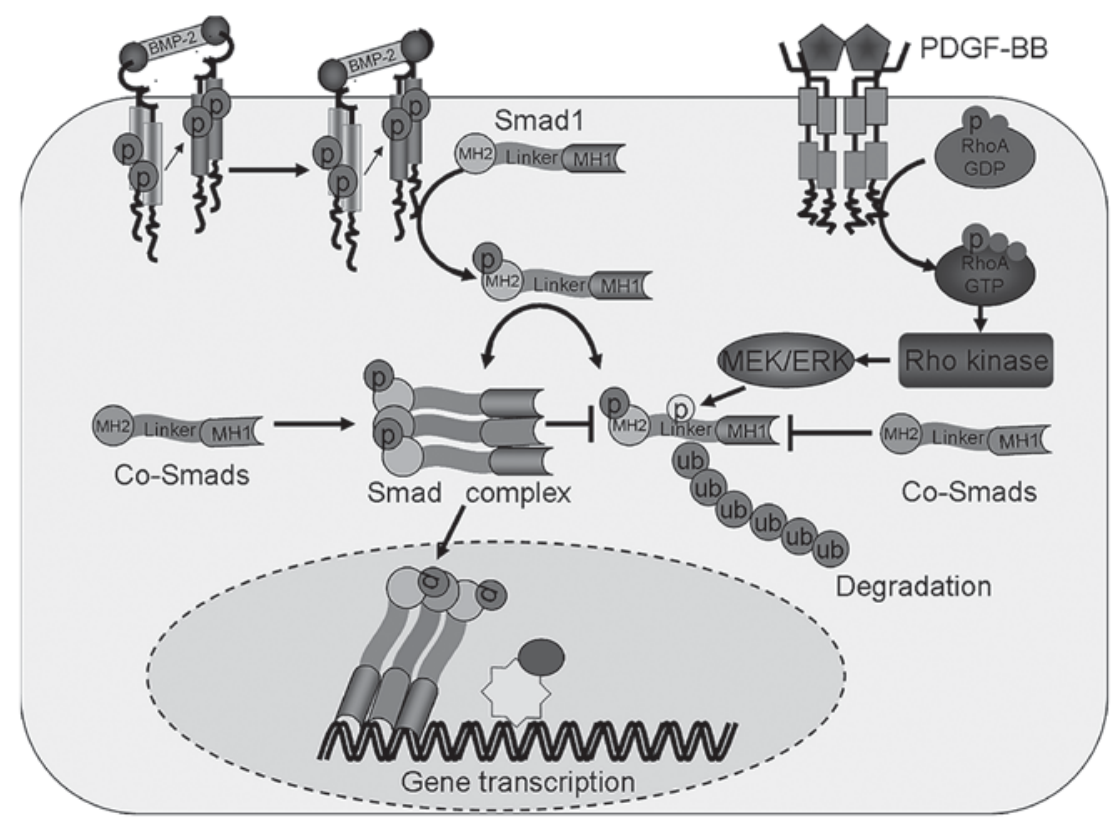

Figure 5. Crosstalk between the Rho kinase and BMP-2 signaling pathways. Crosstalk between the Rho kinase and BMP-2 signaling pathways occur via the phosphorylation of the Smad1 linker region, which leads to the degradation or phosphorylation of its C-terminal, resulting in its translocation into the nucleus. Linker region phosphorylation following the activation of the RHO kinase signaling pathway leads to Smad1 ubiquitination and degradation. The C-terminus phosphorylation of Smad1 induced by BMP-2 leads to nuclear the translocation of this protein. PDGF-BB, platelet derived growth factor-BB; Smad1, mothers against decapentaplegic homolog 1; BMP-2, bone morphogenetic protein 2; p, phosphorylated; ub, ubiquitination; RhoA, transforming protein RhoA; GTP, guanosine triphosphate; GDP, guanosine diphosphate; Co-Smads, common mediator mothers against decapentaplegic homologs; MEK, mitogen-activated protein kinase kinase; ERK, mitogen-activated protein kinase; $\mathrm{MH}$, mad homology domain. 
induced by BMP-2-mediated phosphorylation at Ser463/465. These results suggest a novel molecular mechanism by which the Rho kinase signaling may intercept the BMP signaling and stimulate the proliferation of PAH-PASMCs. This implies that p-Smad1 may be a novel marker or a treatment target in PAH therapy.

Previous studies indicated that the Rho kinase and BMP signaling pathways serve important counter-regulatory roles in pulmonary vascular hypertension (3-7,26-28). As an important molecule, activated Rho kinase pathway participates in the pathogenesis of hypoxia-, monocrotaline- and high pulmonary blood flow-induced $\mathrm{PAH}$; treatment with ROCK inhibitors reduces pulmonary artery pressure and attenuates pulmonary arterial lesions in animal models of PAH $(18,19,26)$. In clinical trials, activated Rho kinase was also detected in patients with PAH, and Rho kinase inhibitor fasudil could decrease pulmonary artery pressure and pulmonary artery resistance $(27,28)$. By contrast, the BMP signaling pathway exhibits meaningful effects on PAH. For example, Luo et al (29) demonstrated that APN suppressed the proliferation of PAH-PASMCs by regulating the AMPK/BMP/Smad pathway. Furthermore, Zhang et al (30) indicated that BMP-2 participates in the regulation of $\mathrm{Ca}\left({ }^{2+}\right)$ signaling in PAH-PASMCs, reducing cell proliferation and migration $(29,30)$. Mutations in BMPR2 or reduced expression levels of BMPR2 and Smad1 were observed in patients with PAH and animal models of this disease (3-7).

Activation of Rho kinase and disruption of BMP signaling both have been demonstrated to promote the proliferation of PASMCs $(31,32)$. In the present study, PDGF-BB stimulated proliferation of PASMCs by interfering with nuclear accumulation of Smad1, suggesting that the Rho kinase signaling and BMP signaling converge on Smad1.

Protein expression levels were determined by western blotting to elucidate the mechanism of PDGF-BB-mediated inhibition of nuclear accumulation of Smad1. PDGF-BB inhibited BMP-2-induced nuclear accumulation of Smad1 through certain mechanisms, inducing degradation. Furthermore, PDGF-BB stimulated the Rho signaling pathway and increased Smad1 phosphorylation at Ser206, leading to the degradation of Smad1 and the interruption of nuclear translocation. Rho kinase has been demonstrated to mediate the mitogenic effect of PDGF-BB on PASMCs in PAH (33). MAPK was demonstrated to prevent nuclear translocation of Smads by phosphorylating the linker region (14). In the present study, ROCK inhibitor or MEK1/2 inhibitor reversed the PDGF-BB-mediated suppression of nuclear translocation of Smad1. It may therefore be hypothesized that PDGF-BB phosphorylates the linker region of p-Smad1 via the Rho kinase/MEK/ERK pathway, leading to its degradation and decreased nuclear translocation. Increased expression levels of $\mathrm{p}$-Smad1 (Ser206) were observed in the PAH-PAMSCs treated with BMP-2 compared with the untreated PAH-PAMSCs; however, treatment with PDGF-BB decreased the expression levels of p-Smad1 (Ser206) compared with untreated cells. PDGF-BB treatment increased p-Smad1 (Ser206), leads to its degradation. However, BMP-2 affected p-Smad1 (Ser463/465), but not $\mathrm{p}$-Smad1 (Ser206). The reason for the increase of p-Smad1 (Ser206) may be that BMP-2 is short-acting and decreases its effect. The results of the present study indicate that Rho kinase phosphorylates $\mathrm{p}$-Smad1 at the linker region and BMP-2 phosphorylate Smad1 at the C-terminal; these two signaling pathways crosstalk at $\mathrm{p}-\mathrm{Smad} 1$ and exert counter regulatory effects on the cell fate. Future studies are required to further confirm this hypothesis, including those to detect the phosphorylation of truncations, which contain different lengths and locations of Smad1.

Rho-kinase appears to be a point of convergence of various signal transduction pathways involved in the mechanism of both primary and secondary PAH $(31,34)$. The results of the present study suggested that suppressed nuclear translocation of Smad1 is one of the mechanisms used by the activated Rho kinase to stimulate the proliferation of PASMCs in PAH. In conclusion, PDGF-BB-activated Rho kinase suppressed BMP-2-induced nuclear translocation $\mathrm{p}$-Smad1 via MEK1/ERK1/2 in rat PASMCs.

\section{Acknowledgements}

Not applicable.

\section{Funding}

The present study was supported by the Foundation for Medical Program of Shandong Province (grant no. 2013WS0220) and the Key Research and Development Plan of Shandong Province (grant no. 2017G006042).

\section{Availability of data and materials}

All data generated or analyzed during this study are included in this published article.

\section{Authors' contributions}

HW performed the experiments; DZ analyzed the data and drafted the manuscript; LL aquired and analyzed the data; WX conceived and designed the present study; FL designed the current study, acquired data, analyzed and interpreted the data, and drafted the manuscript critically for important intellectual content. All authors read and approved the final manuscript.

\section{Ethics approval and consent to participate}

The animal welfare and experimental procedures were performed in accordance with the Guide for the Care and Use of Laboratory Animals, published by the Ministry of Science and Technology of China. The present study was also approved by the Animal Ethics Committee of Shandong University (Shandong, China).

\section{Patient consent for publication}

Not applicable.

\section{Competing interests}

The authors declare that they have no competing interests. 


\section{References}

1. Miyazono K, Kamiya Y and Morikawa M: Bone morphogenetic protein receptors and signal transduction. J Bio chem 147: 35-51, 2010.

2. Sieber C, Kopf J, Hiepen C and Knaus P: Recent advances in bmp receptor signaling. Cytokine Growth Factor Rev 20: 343-355, 2009.

3. Awad KS, West JD, de Jesus Perez V and MacLean M: Novel signaling pathways in pulmonary arterial hypertension. Pulm Circ 6: 285-294, 2015.

4. West J, Austin E, Fessel JP, Loyd J and Hamid R: Rescuing the BMPR2 signaling axis in pulmonary arterial hypertension. Drug Discov Today 19: 1241-1245, 2014.

5. Soubrier F, Chung WK, Machado R, Grünig E, Aldred M, Geraci M, Loyd JE, Elliott CG, Trembath RC, Newman JH and Humbert M: Genetics and genomics of pulmonary arterial hypertension. J Am Coll Cardio 162 (25 Suppl): D13-D21, 2013.

6. Ormiston ML, Upton PD, Li W and Morrell NW: The promise of recombinant BMP ligands and other approaches targeting BMPR-II in the treatment of pulmonary arterial hypertension. Glob Cardiol Sci Pract 2015: 47, 2015.

7. Long L, Ormiston ML, Yang X, Southwood M, Gräf S, MachadoRD Mueller M, Kinzel B, Yung LM, Wilkinson JM, et al: Selective enhancement of endothelial BMPR-II with BMP9 reverses pulmonary arterial hypertension. Nat Med 21: 777-785, 2015

8. Yang J, Li X, Al-Lamki RS, Wu C, Weiss A, Berk J, Schermuly RT and Morrell NW: Sildenafil potentiates bone morphogenetic protein signaling in pulmonary arterial smooth muscle cells and in experimental pulmonary hypertension. Arterioscler Thromb Vasc Biol 33: 34-42, 2013

9. Price LC, Montani D, Tcherakian C, Dorfmüller P, Souza R, Gambaryan N, Chaumais MC, Shao DM, Simonneau G Howard LS, et al: Dexamethasone reverses monocrotaline-induced pulmonary arterial hypertension in rats. Eur Respir J 37: 813-822, 2011.

10. Machado RD, Southgate L, Eichstaedt CA, Aldred MA, Austin ED, Best DH, Chung WK, Benjamin N, Elliott CG, Eyries M, et al: Pulmonary arterial hypertension: A current perspective on established and emerging molecular genetic defects. Hum Mutat 36 1113-1127, 2015.

11. Upton PD and Morrell NW: The transforming growth factor- $\beta$-bone morphogenetic protein type signalling pathway in pulmonary vascular homeostasis and disease. Exp Physiol 98: $1262-1266,2013$

12. Wrana JL: Regulation of smad activity. Cell 100: 189-192, 2000.

13. Sapkota G, Alarcón C, Spagnoli FM, Brivanlou AH and Massagué J: Balancing BMP signaling through integrated inputs into the Smad1 linker. Mol Cell 25: 441-454. 2007.

14. Kretzschmar M, Doody J and Massague J: Opposing bmp and egfsignalling pathways converge on the tgf-beta family mediator smad1. Nature 389: 618-622, 1997.

15. Wilson JL, Yu J, Taylor L and Polgar P: Hyperplastic growth of pulmonary artery smooth muscle cells from subjects with pulmonary arterial hypertension is activated through JNK and p38 MAPK. PLoS One 10: e0123662, 2015.

16. Jin C, Guo J, Qiu X, Ma K, Xiang M, Zhu X and Guo J: IGF-1 induces iNOS expression via the p38 MAPK signal pathway in the anti-apoptotic process in pulmonary artery smooth muscle cells during PAH. J Recept Signal Transduct Res 34: 325-331, 2014.

17. Ziino AJ, Ivanovska J, Belcastro R, Kantores C, Xu EZ, Lau M, McNamara PJ, Tanswell AK and Jankov RP: Effects of rho-kinase inhibition on pulmonary hypertension, lung growth, and structure in neonatal rats chronically exposed to hypoxia. Pediatr Res 67 177-182, 2010.

18. Yasuda T, Tada Y, Tanabe N, Tatsumi K and West J: Rho-kinase inhibition alleviates pulmonary hypertension in transgenic mice expressing a dominant-negative type II bone morphogenetic protein receptor gene. Am J Physiol Lung Cell Mol Physiol 301: L667-L674, 2011.

19. Jasińska-Stroschein M, Owczarek J, Sołtysiak U and Orszulak-Michalak D: Rosuvastatin intensifies the beneficial effects of rho-kinase inhibitor in reversal of monocrotaline-induced pulmonary hypertension. Arch Med Sci 12: 898-905, 2016.
20. Liu Y, Ren W, Warburton R, Toksoz D and Fanburg BL: Serotonin induces Rho/ROCK-dependent activation of Smads 1/5/8 in pulmonary artery smooth muscle cells. FASEB J 23: 2299-2306, 2009.

21. Chen XY, Dun JN, Miao QF and Zhang YJ: Fasudil hydrochloride hydrate, a rho-kinase inhibitor, suppresses 5-hydroxytryptamine-induced pulmonary artery smooth muscle cell proliferation via jnk and erk1/2 pathway. Pharmacology 83 : 67-79, 2009.

22. Tanabe T, Furuya H, Kanemoto N, Goto Y and Hata J: Experimental study on monocrotaline induced pulmonary hypertensive rats. (1) Effect of long-term injection of immunosuppressant. Tokai J Exp Clin Med 6: 41-48, 1981

23. Li F, Xia W, Li A, Zhao C and Sun R: Long-term inhibition of Rho kinase with fasudil attenuates high flow induced pulmonary artery remodeling in rats. Pharmacol Res 55: 64-71, 2007.

24. The Ministry of Science and Technology of the People's Republic of China: Guidance Suggestions for the Care and Use of Laboratory Animals, 2006. http://www.most.gov.cn/fggw/zfwj/ zfwj2006/200609/t20060930_54389.htm. Accessed September 30, 2006.

25. Amano M, Nakayama M and Kaibuchi K: Rho-kinase/ROCK: A key regulator of the cytoskeleton and cell polarity. Cytoskeleton (Hoboken) 67: 545-554, 2010

26. Gupta V, Gupta N, Shaik IH, Mehvar R, McMurtry IF, Oka M, Nozik-Grayck E, Komatsu M and Ahsan F: Liposomal fasudil, a rho-kinase inhibitor, for prolonged pulmonary preferential vasodilation in pulmonary arterial hypertension. J Control Release 167: 189-199, 2013.

27. Xiao JW, Zhu XY, Wang QG, Zhang DZ, Cui CS, Zhang P, Chen HY and Meng LL: Acute effects of Rho-kinase inhibitor fasudilon pulmonary arterial hypertension in patients with congenital heart defects. Circ J 79: 1342-1348, 2015.

28. Jiang X, Wang YF, Zhao QH, Jiang R, Wu Y, Peng FH, Xu XQ, Wang L, He J and Jing ZC: Acute hemodynamic response of infused fasudil in patients with pulmonary arterial hypertension: A randomized, controlled, crossover study. Int J Cardiol 77: 61-65, 2014.

29. Luo L, Zheng W, Lian G, Chen H, Li L, Xu C and Xie L: Combination treatment of adipose-derived stem cells and adiponectin attenuates pulmonary arterial hypertension in rats by inhibiting pulmonary arterial smooth muscle cell proliferation and regulating the AMPK/BMP/Smad pathway. Int J Mol Med 41: $51-60,2018$

30. Zhang Y, Lu W, Yang K, Xu L, Lai N, Tian L, Jiang Q, Duan X, Chen $\mathrm{M}$ and Wang J: Bone morphogenetic protein 2 decreases TRPC expression, store-operated $\mathrm{Ca}(2+)$ entry, and basal $[\mathrm{Ca}(2+)] \mathrm{i}$ in rat distal pulmonary arterial smooth muscle cells. Am J Physiol Cell Physiol 304: C833-C843, 2013.

31. Watanabe H: Rho-kinase activation in patients with pulmonary arterial hypertension. Circ J 73: 1597-1598, 2009.

32. Morrell NW: Role of bone morphogenetic protein receptors in the development of pulmonary arterial hypertension. AdvExp Med Biol 661: 251-264, 2010.

33. Kamiyama M, Utsunomiya K, Taniguchi K, Yokota T, Kurata H, Tajima $\mathrm{N}$ and Kondo K: Contribution of Rho A and Rho kinase to platelet-derived growth factor-BB-induced proliferation of vascular smooth muscle cells. J Atheroscler Thromb 10: 117-123, 2003.

34. Nunes KP, Rigsby CS and Webb RC: Rhoa/rho-kinase and vascular diseases: What is the link? Cell Mol Life Sci 67: 3823-3836, 2010

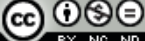

This work is licensed under a Creative Common Attribution-NonCommercial-NoDerivatives 4.0 International (CC BY-NC-ND 4.0) License. 